\title{
Percepção e avaliação dos alunos do curso de Medicina de uma escola médica pública sobre a importância do estágio em saúde da família na sua formação
}

PALAVRAS-CHAVE:

Saúde da Familia;

Atenção Primária à Saúde;

Educação Médica;

Estudantes de Medicina

Saúde na Comunidade.

Saúde coletiva

Internato e residência

Capacitação em Serviço

KEYWORDS

Family Health;

Primary Health Care

Medical Education;

Medical Students;

Community Health.

Publichealth

Internship and Residency

Inservice Training

Recebido em: 15/02/2007

Reencaminhado em: 02/08/2007

Aprovado em: 11/10/2007

\section{The importance of the Family Medicine experience from the viewpoint of students of a public medical school}

Maria Angélica de Figueiredo Campos Aldaísa Cassanho Forster ${ }^{1}$

\section{RESUMO}

Na Faculdade de Medicina de Ribeirão Preto da Universidade de São Paulo (FMRP-USP), o ensino da Atenção Primária (AP) com enfoque em medicina de família no primeiro ano de internato se iniciou em 1997. A estratégia de ensino consiste em inserir o aluno em equipes de saúde da família para vivenciar as práticas na realidade do Sistema Único de Saúde. As práticas programadas foram consulta médica individual, seguimento de famílias, visitas domiciliares, atividades de grupos e discussões de casos individuais e de famílias. Foram enfatizadas a prevenção de doenças e a promoção da saúde, bem como a vivência com o usuário, buscando-se a assistência integral. O objetivo deste trabalho foi estudar a contribuição do estágio no Programa de Saúde da Família (PSF) para a formação dos alunos do quinto ano do curso de Medicina da Faculdade de Medicina de Ribeirão Preto da Universidade de São Paulo (FMRP-USP), em AP, tendo 103 alunos respondido a um questionário estruturado aplicado antes e depois do estágio. Segundo o aluno, o estágio contribuiu de forma positiva para a sua formação, principalmente nos aspectos de integração com a equipe de saúde, humanização e visão dos principais princípios da saúde da família e da AP, tais como trabalho em equipe, longitudinalidade, acessibilidade e atuação na prevenção. Após o estágio, o aluno passou a dar mais importância aos aspectos sociais e econômicos do paciente e a avaliá-lo como um ser biopsicossocial

\section{ABSTRACT}

Primary Care (PC) centered on family medicine started to be taught at the Faculty of Medicine of Ribeirão Preto, São Paulo University (FMRP-USP), in 1997. The teaching strategy consists in introducing students into Family Health teams, where they are encouraged to participate in the routine Family Practice, taking part in group activities, team meetings, household visits, and discussions of individual and family cases. Disease prevention, health promotion and the relationship with the user of the care delivery system are emphasized in an effort to 
deliver comprehensive care. The objective of this study was to evaluate to which extent, in the viewpoint of 5th year medical students of FMRP-USP, the Family health experience contributed to their Primary Care training. A structured questionnaire was applied to 103 students before and after the training. The training contributed positively to the education of the students, especially as refers to the aspects integration with the health team, patientcenteredness, and awareness of the main principles of family medicine such as team work, long-term care, accessibility and preventive care. After the training the students passed do give more importance to the social and economic aspects of the individual and to see the patient as bio-psychosocial being.

\section{INTRODUÇÃO}

No Brasil, o processo de ensino na maioria das faculdades de Medicina ainda privilegia o aprendizado em centros terciários, voltados para cuidar de problemas de maior complexidade. Nesse contexto, constata-se que o curso médico prioriza a formação especializada e o aprendizado dessas especialidades, sendo organizado de forma fragmentada, o que impede o aluno de ter uma visão geral do paciente. Esse perfil de médico egresso das faculdades de Medicina não corresponde àquele desejado pelo mercado de trabalho do Sistema Único de Saúde e do Programa de Saúde da Família (PSF).

Assim, tendo o hospital universitário como cenário principal do aprendizado médico, o aluno acompanha, na maioria das vezes, pacientes com patologias já instaladas, com um nível de gravidade alto ou com seqüelas, e realiza uma atuação pontual no doente, que depois será acompanhado por outros médicos e alunos, resultando numa visão fragmentada do paciente. Nesse processo, a visão que o aluno adquire é a de que sua atuação no processo saúde-doença consiste apenas em tratar patologias ou suas complicações. Porém, sabe-se que, na vida profissional do graduado, seu campo de trabalho não está restrito ao setor terciário; pelo contrário, a maioria dos postos de trabalho está nos setores primário e secundário.

O Programa de Saúde da Família (PSF), introduzido na década de 1990 pelo Ministério da Saúde, criou um extenso campo de trabalho para o médico. Para seu sucesso, contudo, são necessários médicos com características particulares: generalistas, capazes de se inserir em um bairro, lidar com indicadores epidemiológicos, com cobertura populacional, promover práticas de vigilância à saúde e se integrar com os demais profissionais da saúde ${ }^{1}$.
Diante dessa realidade, as universidades vêm buscando se adequar às Diretrizes Curriculares do curso de Medicina, mediante a expansão do ensino para o nível primário de atenção, ou seja, Centros de Saúde ou Unidades de Saúde da Família. Nestes, o aluno inicia um contato precoce com a comunidade em que irá atuar. Além disso, os currículos estão sendo reorganizados para que os estudantes tenham um contato longitudinal com a atenção básica, estagiando em determinada Unidade de Saúde durante todo o curso. Inicialmente, os alunos têm noções de territorialização, verificação das condições de saúde na comunidade, conhecimentos da rotina de um Centro de Saúde e, ao longo do curso, desenvolvem as habilidades clínicas, ficando incumbidos de atender determinadas famílias, desenvolver ações de prevenção e participar das atividades das equipes.

A consideração da atenção primária como nível adequado de formação profissional para os alunos de Medicina é determinada pelo fato de que, na comunidade, o aluno está numa posição privilegiada para aprender as atividades preventivas, compreender a realidade do processo saúdedoença e sua abordagem holística, assim como a complexidade e importância da relação médico-paciente e da longitudinalidade da atenção, além de compreender a abordagem das doenças prevalentes, tanto agudas como crônicas ${ }^{2}$.

A atenção primária aborda os problemas mais freqüentes da comunidade, oferecendo serviços de prevenção, cura e reabilitação para maximizar a saúde e o bem-estar. Ela integra a atenção quando há mais de um problema de saúde e lida com o contexto em que a doença se desenvolve. É a atenção que organiza e racionaliza o uso de todos os recursos, tanto básicos como especializados ${ }^{3}$.

\section{O ENSINO EM ATENÇÃO PRIMÁRIA NA FMRP}

Na Faculdade de Medicina de Ribeirão Preto da Universidade de São Paulo (FMRP-USP), o ensino da atenção primária com enfoque em medicina de família se iniciou em 1997, com as disciplinas Medicina Comunitária I e Estágio Integrado em Centros de Saúde. Esse módulo foi introduzido no quinto ano de graduação em Medicina, com duração de oito semanas ${ }^{4}$, e suas atividades são realizadas no Centro de Saúde Escola (CSE) da FMRP-USP, em suas Unidades Distrital e Básicas, e, desde 2001, também nos Núcleos de Saúde da Família (NSF) I, III, IV e V.

\section{Os objetivos do estágio no PSF são:}

(1) Inserir o aluno do quinto ano de Medicina nas práticas em serviços de Atenção Primária e Saúde da Família, em regime de internato, de forma que possa: 
(2) Vivenciar o papel de médico de Atenção Primária (APS) e de Família em equipe multiprofissional e em serviços integrados com os níveis especializados do SUS;

(3) Conhecer os problemas de saúde de alta prevalência na comunidade;

(4) Participar das ações de vigilância epidemiológica em uma área distrital;

(5) Apreender a concepção moderna da Atenção Primária: (co-responsabilização do paciente e da família, integralidade das práticas de saúde, intersetorialidade).

No estágio nos Núcleos de Saúde da Família, a cada oito semanas um grupo de 20 alunos, em média, é dividido em subgrupos de cinco ou seis para cada Núcleo, onde estagiam durante um período por dia, de segunda a quintafeira, sendo a sexta-feira destinada a seminários e aulas teóricas sobre temas mais relevantes em atenção primária. Os alunos são supervisionados pelos médicos das equipes, que são especialistas em medicina de família e comunidade, e pelos docentes da disciplina. Suas atividades são:

- participar das visitas domiciliares com os membros da equipe;

- participar das atividades de educação em saúde, como grupo de diabéticos, hipertensos, reeducação alimentar, gestantes, e de vivências;

- participar das reuniões de educação continuada da equipe, nas quais, a cada semana, dois alunos e um residente são responsáveis por desenvolver um tema;

- participar das discussões de casos de família, semanalmente;

- vigilância à saúde, que consiste no estudo da prevalência de determinadas doenças na área de abrangência, sob orientação do docente, e elaboração de um plano das ações de prevenção e tratamento a ser desenvolvido pelo NSF;

- consultas de pacientes e discussão de famílias seguidas no NSF.

Nesse estágio, é promovida a integração do aluno com a equipe de PSF e a sua participação na rotina da equipe por meio dos grupos, reuniões de equipe, visitas domiciliares, discussões de casos individuais e de família, com a finalidade de contribuir para a sua formação multiprofissional. No atendimento ambulatorial, são enfatizadas a prevenção e a promoção da saúde e a avaliação do paciente, buscando a assistência integral. No atendimento dos outros membros de uma mesma família, é designado o mesmo aluno, para que ele compreenda a importância da visão do paciente no contexto familiar, entendendo como é o relacionamento deste com os demais membros da família e como esse fator pode interferir no processo saúde-doença.

\section{OBJETIVOS}

O objetivo deste trabalho foi estudar a contribuição do estágio no PSF para a formação dos alunos de Medicina do quinto ano do Curso de Ciências Médicas da Faculdade de Medicina de Ribeirão Preto da Universidade de São Paulo (FMRP-USP). Os objetivos específicos foram: conhecer a avaliação do aluno sobre a importância e contribuição de cada aspecto do estágio; caracterizar a percepção do aluno sobre a contribuição do estágio na relação dele com o paciente/usuário do serviço de saúde; e identificar os princípios da APS vivenciados pelo aluno durante o estágio.

\section{METODOLOGIA}

Este trabalho se classifica como uma pesquisa quantitativa. Os sujeitos do estudo eram alunos do quinto ano do Curso de Ciências Médicas da Faculdade de Medicina de Ribeirão Preto-USP matriculados na disciplina de Medicina Comunitária I em 2005. Foram entrevistados 103 alunos, de janeiro a outubro de 2005.

O primeiro questionário continha nove perguntas e foi aplicado no primeiro dia do estágio; o segundo continha 17 perguntas, incluindo as do questionário anterior, e foi aplicado no último dia do estágio a todos os alunos simultaneamente. As perguntas eram fechadas e abertas.

\section{As perguntas do questionário se referiam a:}

(1) dados pessoais;

(2) vivência no Núcleo de Saúde da Família, contemplando os elementos de integração com a equipe e novas práticas no trabalho em equipe multiprofissional;

(3) avaliação do grau de satisfação, da importância dada pelo aluno ao estágio e da influência desse estágio na formação do aluno (relação médico-paciente e atuação na família). Foi sugerida ao aluno uma classificação em escores para expressar sua apreciação sobre cada assunto (nada importante, pouco importante, moderadamente importante, bastante importante e muito importante) (Anexos 1 e 2).

A análise consistiu na distribuição das freqüências das respostas às questões fechadas, com emprego do programa estatístico Epiinfo 2002. As respostas às questões abertas foram analisadas quanto à freqüência e à importância dada na justificativa pelos alunos às respostas fechadas. 


\section{RESULTADOS E DISCUSSÃO}

Cento e três alunos responderam os questionários, sendo $53,3 \%$ do sexo masculino e $41,7 \%$ do sexo feminino. Segundo a faixa etária, a maioria dos alunos tinha entre 21 e 24 anos, sendo a média de 23,4 anos, e 93,1\% eram procedentes de cidades do estado de São Paulo.

Primeiramente, os alunos avaliaram a importância do estágio em saúde da família na sua formação, classificando-o em nada, pouco, moderadamente, bastante ou muito importante.

No primeiro questionário, essa pergunta foi respondida por 102 alunos, e a maioria o classificou como bastante importante $(48 \%)$ ou muito importante $(27,5 \%)$. O segundo questionário foi respondido por 102 alunos, e houve pequena variação na freqüência das respostas.

Na pergunta seguinte, os alunos foram indagados se consideravam que o estágio em saúde da família poderia contribuir para a sua formação médica. No questionário inicial, dos 73 alunos que justificaram a resposta, 39,7\% indicaram como principal contribuição o conhecimento e atuação em atenção primária, 20,6\% citaram o contato com questões socioeconômicas e familiares, e 15,1\% indicaram a prática de medicina integral.

Ao final do estágio, 59 alunos justificaram sua resposta considerando que a contribuição se deveu ao conhecimento e atuação em atenção primária $(30,5 \%)$ e à prática da medicina integral (22\%). Para 5,1\% desses alunos, o estágio contribuiu para que melhorassem a relação médico-paciente.

Sobre a inserção do estágio no quinto ano, a maioria dos 103 alunos - $86,4 \%$ e $87,4 \%$ - respondeu ao primeiro e segundo questionários, respectivamente, julgando-a adequada, pois sua formação no ciclo básico e clínico havia sido completada. Em contraste, 15\% e 16\%, respectivamente, sugeriram que o estágio deveria anteceder o internato. Porém, conforme as Diretrizes Curriculares do Curso de Medicina, o curso deve "inserir o aluno precocemente em atividades práticas relevantes para a sua futura vida profissional". Por isso, considerase importante a atuação do aluno nas atividades dos Núcleos de Saúde da Família desde os primeiros anos de curso, para que se construa um vínculo duradouro com a equipe e a comunidade ${ }^{5}$.

A questão: "Você considera que a vivência nos NSF irá possibilitar uma compreensão real do PSF?" foi respondida por 103 alunos. No questionário inicial, 40,8\% responderam afirmativamente, e esse número aumentou para $65 \%$ após o estágio. Segundo os estudantes, essa compreensão foi possível porque eles participaram de todas as atividades desenvolvidas nos Núcleos.

No primeiro questionário, 11 dos 17 alunos e no segundo $36,4 \%$ dos 22 alunos que responderam "não" comentaram que o vínculo com a Universidade diminui o grau de comparabilidade dos Núcleos com as unidades típicas do PSF. Os Núcleos de Saúde da Família realmente são ambientes distintos, voltados não só para a assistência, mas especialmente para o ensino, e não reproduzem exatamente uma Unidade de Saúde da Família, pois possuem supervisão contínua de uma Universidade. É bom que o aluno tenha percebido essa diversidade e que possa optar no futuro por um local de trabalho que contemple as ações vivenciadas em seu campo de estágio.

\section{As perguntas seguintes corresponderam ao segundo questionário, aplicado no final do estágio.}

$\mathrm{Na}$ pergunta seguinte, os alunos classificaram as atividades desenvolvidas no estágio de acordo com a importância para a sua formação, em nada, pouco, moderadamente, bastante ou muito importante. As atividades listadas foram: atuação médica em equipe, assistência médica ampliada para a família, visita domiciliar, consultas médicas dos membros das famílias, atividades educativas como grupos, palestras, ações junto à comunidade, discussão de casos de família, seminários e discussão de casos e atuação em saúde mental.

As atividades classificadas como bastante ou muito importantes por mais de $80 \%$ dos alunos foram: discussão e atuação em saúde mental, atuação em equipe e assistência à família. As classificadas por menos de $60 \%$ dos alunos como bastante ou muito importantes foram: visita domiciliar, seminários e discussão de casos de família.

As atividades com mais respostas "nada" e "pouco importante" foram: visita domiciliar $(19,6 \%)$, seminários $(16,8 \%)$ e discussão de casos de família $(14,7 \%)$.

A maioria dos alunos cuja justificativa apresentada foi "a discussão e atuação em saúde mental" (61,5\%) explicou que o motivo de sua importância é a alta freqüência de transtornos psiquiátricos observada na demanda do serviço de saúde. Uma percentagem menor citou que ela possibilita o seguimento dos pacientes no próprio NSF, "desafogando" os serviços especializados em saúde mental $(15,4 \%)$, e permite práticas antes não vivenciadas no curso $(11,5 \%)$. O estágio é um importante momento para a abordagem de transtornos psiquiátricos 
de alta prevalência, como transtornos depressivos e de ansiedade, e o médico, não somente o psiquiatra, deve saber detectá-los precocemente e conduzi-los juntamente com a equipe. No estágio, o aluno também aprende a distinguir os casos que podem ser seguidos na atenção primária daqueles que devem ser encaminhados para atendimento nos setores secundário e terciário.

Os alunos também reconheceram como importante a atuação em equipe, porque promove a integração com outros profissionais $(36,7 \%)$, além de aumentar a adesão do paciente ao tratamento, e a assistência ampliada à família, porque proporciona maior resolutividade $(42 \%)$.

Em relação à visita domiciliar, a resposta foi que não é atribuição do médico ou que é pouco efetiva. A experiência discente é pequena com a visitação domiciliar, e sua inserção somente nas atividades do internato médico também contribui para essa inadequação. A visita domiciliar é realizada com o médico, a enfermeira, o auxiliar de enfermagem e, na maioria das vezes, com o Agente Comunitário de Saúde (ACS). Como o objetivo da visita do ACS com o aluno não é realizar intervenções médicas, isso pode desmotivar o aluno, que não encara a visita domiciliar como parte do rol de práticas médicas.

Para as outras respostas, poucos alunos ofereceram justificativas, destacando-se o item "discussão de casos de família", que 10\% de 30 alunos consideraram "invasão da vida alheia". A discussão de casos de família aborda principalmente problemas sociais e familiares e de acesso aos serviços de saúde. Talvez o aluno encare esse fato como invasão da privacidade dos pacientes e das famílias. Esse tipo de discussão - que não aborda somente aspectos biológicos e permite que a equipe trace um plano de ação e defina conjuntamente como será conduzido cada caso - é bastante distinto da tradicional discussão de casos clínicos conhecida pelo aluno.

Para $49,5 \%$ e $82,5 \%$ dos alunos, o tempo de estágio foi adequado para a sua inserção nas atividades da equipe de Saúde da Família - respostas dadas ao início e término da disciplina, respectivamente.

A questão seguinte enfocou se as condições do estágio possibilitaram uma boa integração entre os alunos e a equipe do NSF. A grande maioria (91,3\%) afirmou que sim. Embora o total de alunos que justificaram tenha sido pequeno (25 alunos), a principal explicação, na visão destes, foi a receptividade proporcionada pela equipe (36\%). Essa receptividade percebida pelo estudante pode estar refletindo o fato de existir um ambiente de formação nos Núcleos de Saúde da Família (graduação, pós-graduação senso lato e educação permanente), graças à orientação acadêmica implementada pela USP em seus serviços de extensão. Essa interação aluno-equipe se torna uma rotina nas unidades, e o aluno se sente parte da equipe, participando ativamente das atividades de educação à saúde.

A pergunta seguinte - "Você considera que o trabalho em equipe contribui para a continuidade do seguimento do paciente? De que forma?" - foi respondida por 63 estudantes, sendo que todos afirmaram "sim" na primeira parte da questão. As razões mais citadas foram que o trabalho em equipe possibilita melhor vínculo com o paciente $(27 \%)$ e promove a abordagem multiprofissional $(27 \%)$. Isso mostra como essa vivência do aluno no dia-adia do trabalho em equipe possibilita que ele avalie os benefícios da construção desse vínculo para as famílias que ele acompanha.

Os alunos responderam que observaram mudanças em sua forma de atender $(68 \%)$, sendo a principal delas a visão que passaram a apresentar do paciente como um ser biopsicossocial. Outra mudança muito favorável percebida pelo aluno, e citada por 18,3\% dos 60 que justificaram, foi maior habilidade, segurança e paciência (Tabela 6). Durante esse convívio mais próximo com as famílias, o aluno percebe a complexidade e a variedade dos fatores que determinam a qualidade de vida daquela população. Ao longo das visitas, reuniões, atendimentos e da integração com a equipe do PSF, ele apreende como agir para promover a saúde do paciente e das famílias que ele acompanha e, pelo menos a curto e médio prazos, visualiza algum resultado alcançado. Como conseqüência, o graduando obtém mais segurança nas ações médicas que realiza e percebe que o paciente não deve ser avaliado somente pelos distúrbios orgânicos que apresenta, pois não são apenas esses os fatores determinantes da saúde e da doença que interferem na qualidade de vida dele. O estágio possibilita que o estudante reflita sobre o quadro de saúde de determinadas famílias que receberam suas ações, de outros alunos e da equipe, quadro este que depende da intervenção sobre outros setores além da saúde (intersetorialidade na Atenção Primária e Saúde da Família). Apesar da frustração que daí resulta, é mais uma oportunidade para que o aluno compreenda as limitações das ações em saúde, especialmente das ações médicas (Tabela 1). 


\section{TABELA 1}

\section{Percepção do aluno sobre as modificações na sua forma de atender o paciente durante o estágio em saúde da família, 2005}

\begin{tabular}{clc}
\hline Mudanças no atendimento percebidas pelo aluno & $\mathrm{N}$ & $\%$ \\
\hline Mais atenção ao aspecto biológico, psíquico e social & 33 & 61,1 \\
Mais habilidade, segurança, paciência & 11 & 20,4 \\
Melhor relação médico-paciente & 7 & 12,9 \\
Prática do seguimento periódico do paciente & 3 & 5,6 \\
\hline Total & 54 & 100,0 \\
\hline
\end{tabular}

A questão seguinte foi: "A atenção básica à saúde apresenta alguns princípios para ser desenvolvida. Você considera que o estágio em Saúde da Família possibilitou atividades de assistência que atendiam quais princípios?". Consultando a lista dos princípios, mais de $80 \%$ dos respondentes avaliaram bem a acessibilidade, a longitudinalidade, o trabalho em equipe e a atuação na prevenção (Tabela 2).
Mesmo que nas perguntas anteriores o aluno tenha enfatizado o vínculo paciente-equipe que vivenciou no estágio, ele avaliou como pouco vivenciada a adesão ao tratamento. A resolutibilidade, também pouco observada entre os princípios, explica-se pela curta duração do estágio. Contudo, o atendimento satisfatório da demanda do serviço no momento de sua expressão e o seguimento dos usuários se fazem a médio ou longo prazo.

TABELA 2

Avaliação dos princípios da atenção básica à saúde vivenciados pelo aluno no estágio em saúde da família, 2005

\begin{tabular}{lccc}
\hline \multicolumn{1}{c}{ Princípios } & \multicolumn{1}{c}{ Sim } & \multicolumn{1}{c}{ Não } & Não sabe \\
\hline Acessibilidade & $100(97,1 \%)$ & $3(2,9 \%)$ & 0 \\
Longitudinalidade & $96(94,1 \%)$ & $5(4,9 \%)$ & $1(1 \%)$ \\
Trabalho em equipe & $92(89,3 \%)$ & $10(9,7 \%)$ & $1(1 \%)$ \\
Atuação na prevenção & $89(86,4 \%)$ & $10(9,7 \%)$ & $4(3,9 \%)$ \\
Visão biopsicossocial do & $80(79,2 \%)$ & $13(12,9 \%)$ & $8(7,9 \%)$ \\
paciente & & & \\
Resolutibilidade & $64(66,0 \%)$ & $24(24,7 \%)$ & $9(9,3 \%)$ \\
Adesão do paciente & $57(55,9 \%)$ & $30(29,4 \%)$ & $15(14,7 \%)$ \\
\hline
\end{tabular}

\section{CONCLUSÃO}

Os alunos reconhecem a importância do estágio em Saúde da Família e a contribuição deste para a sua formação. No início do estágio, consideraram que os aspectos mais importantes para a formação foram: conhecimento e atuação em atenção primária, contato com questões socioeconômicas e familiares, e a prática de uma medicina integral. No término do estágio, ao avaliarem que aspectos foram mais importantes para a formação, os alunos selecionaram os mesmos aspectos considerados no início do estágio. Isto demonstra que o estágio conseguiu satisfazer aos anseios dos alunos quanto à vivência no PSF.

Os alunos valorizaram práticas que não realizam comumente em nenhum outro estágio e que contribuem para uma compreensão mais abrangente da saúde na comunidade em que atuam, como discussão de casos voltada para a saúde mental e atuação em saúde mental na Atenção Primária, atuação em equipe multidisciplinar e assistência centrada na família.

Observou-se que há uma boa integração entre as equipes de saúde da família e os alunos. A receptividade dessas equipes foi um fator bem avaliado pelos alunos e, certamente, contribui para inserir os alunos nas equipes e motivá-los para o estágio.

O graduando entende que o trabalho em equipe é necessário, pois possibilita um vínculo melhor com o paciente e promove a abordagem multiprofissional.

Quanto a possíveis mudanças percebidas pelos alunos em sua forma de atender o paciente ao final do estágio, 
concluiu-se que este provocou mudanças na maioria dos alunos, que passaram a compreender a abordagem do paciente como um ser biopsicossocial. Além disso, proporcionou aquisições importantes para o exercício da profissão médica: maior habilidade, segurança e paciência no atendimento aos pacientes.

O estágio possibilitou que os alunos vivenciassem os princípios essenciais na atenção básica, principalmente acessibilidade, longitudinalidade, trabalho em equipe e atuação na prevenção. Os princípios menos vivenciados pelos alunos foram adesão do paciente e resolutibilidade, que, por serem alcançados em longo prazo, precisariam de maior tempo de vivência do aluno no estágio.

O estágio foi bem avaliado em vários aspectos, e a vivência numa equipe do PSF resultou em ganhos para a formação do aluno e na visão da importância do PSF na atenção básica, na promoção da saúde nas comunidades e na humanização da relação entre médico e paciente.

Este estudo forneceu subsídios para o aperfeiçoamento do estágio e mostrou as principais mudanças positivas que essa vivência produziu na formação médica do aluno. Espera-se que esse futuro médico valorize o aspecto biopsicossocial no paciente e em sua família, bem como o trabalho em equipe, de forma longitudinal, contínua e resolutiva. Além disso, é necessário inserir esse aluno cada vez mais precocemente no trabalho desenvolvido pelas equipes de saúde da família.

\section{REFERÊNCIAS}

1. Moretti R. A educação médica diante das necessidades sociais em saúde. Rev Bras Educ Méd 2005; 29(2): 136-146.

2. Pitz PB, Vicente VC. La medicina de família como disciplina acadêmica y la atención primaria como entorno de aprendizaje. Atención Primaria 2004; 34(8): 433-6.

3. Starfield B. Atenção primária: equilíbrio entre necessidades de saúde, serviços e tecnologia. Brasília: Unesco; 2002. p. 28.

4. Forster AC, Passos ADC, Dal-Fabbro AL, Laprega MR. Transformation and trends in preventive and social medicine education at the undergraduate level in a Brazilian medical school. Gac Sanit 2001; 15(6): 519-22.

5. Lampert JB. Tendências de mudanças na formação médica no Brasil. Rio de Janeiro; 2002 Doutorado [Tese] - Escola Nacional de Saúde Pública.

\section{CONFLITOS DE INTERESSE}

Declarou não haver

\section{ENDEREÇO PARA CORRESPONDÊNCIA}

Maria Angélica de Figueiredo Campos

Universidade de São Paulo

Faculdade de Medicina de Ribeirão Preto Av. Bandeirantes, 3900 - Campus da USP

CEP: 14049-900 Ribeirão Preto - São Paulo

E-mail:mariamed@bol.com.br 